\title{
Gold Nanoparticles-Enhanced Amperometric Tyrosinase Biosensor Based on Three-Dimensional Sol-Gel Film-Modified Gold Electrodes
}

\author{
Xianrui LI,* Tiankun REN,** Na WANG,* and Xueping JI*广 \\ *Department of Medical Chemistry, Hebei Medical University, Shijiazhuang 050017, P. R. China \\ **School of Pharmaceutical Sciences, Hebei Medical University, Shijiazhuang 050017, P. R. China
}

\begin{abstract}
An amperometric biosensor for the determination of catechol was developed by immobilizing tyrosinase (tyr) on gold nanoparticles (AuNPs) and a (3-mercaptopropyl)-trimethoxysilane (MPTS) sol-gel three-dimensional network film-modified gold electrode. The AuNPs self-assembled in a sol-gel network provided an excellent microenvironment for an enzymatic reaction between tyrosinase and the substrate. It was found that the AuNPs could significantly affect the electron-transfer kinetics of the enzyme reaction, and remarkably enhance the electrochemical reduction of the corresponding $o$-quinones at the electrode surface. The nanostructured electrode showed high sensitivity $\left(306.7 \mathrm{~mA} \mathrm{M}^{-1} \mathrm{~cm}^{-2}\right)$ toward catechol, with an amperometric detection limit of $0.56 \mu \mathrm{M}$. The catalytic current of the biosensor was linear with the catechol concentration ranging from 1.7 to $96 \mu \mathrm{M}$ with a correlation coefficient of 0.9992 . The proposed biosensor exhibited a short response time, good anti-interferent ability, and excellent operational and storage stability.
\end{abstract}

(Received December 10, 2012; Accepted February 21, 2013; Published April 10, 2013)

\section{Introduction}

The determination of phenolic compounds has attracted considerable attention in the analytical chemistry field because of their high toxicity and detrimental effects to human being health. At present, an electrochemical biosensor has been proven to be an accurate and versatile analytical method for the detection of phenolic compounds, due to such advantages as high selectivity, relatively low cost, and the miniaturization and automation. ${ }^{1-3}$ More recently, various supporting materials, such as graphite composite, ${ }^{4}$ conducting polymer, ${ }^{5,6}$ sol-gel films, ${ }^{7,8}$ and self-assembled monolayers (SAMs), ${ }^{9}$ have been successfully utilized to immobilize enzymes for the construction of a tyrosinase (tyr)-based electrochemical biosensor. Among them, sol-gel film offers a better biointerface for the immobilization of enzymes. The encapsulated enzymes retain the same functionality in comparison with their free-enzymes. However, the apparent activity of the entrapped enzymes is often hindered by the diffusion of the analyte and the product in sol-gel matrices, ${ }^{10,11}$ especially for a larger dimension analyte and product, due to a larger diffusion resistance.

To achieve a highly sensitive biosensor, the signal amplification and the stable immobilization of the enzymes on the transducer surface are of considerable importance. In recent decades, metal nanoparticles have been successfully utilized to improve some biomolecular immobilization and bioassay methodologies due to their unique chemical and physical properties. ${ }^{12,13}$ Especially, gold nanoparticles (AuNPs) have been widely used in the fabrication interface of biosensors to enhance their

$\dagger$ To whom correspondence should be addressed.

E-mail: xpji03@yahoo.com.cn electronic, chemical, and electrochemical properties. ${ }^{14,15}$ The unique properties of AuNPs provide a suitable microenvironment for the immobilization of biomolecules and to facilitate electron transfer. ${ }^{16}$

The integration of metal nanomaterials and a sol-gel membrane has been demonstrated to be one of the powerful and versatile tools to improve the performance of the interface, and to enhance the sensitivity of biosensors. Currently, a (3-mercaptopropyl)trimethoxysilane (MPTS) sol-gel-derived three-dimensional network is attractive in the development of sensing devices. The thiol tail groups of an MPTS three-dimensional network is able to be covalently bound onto the surface of Au through cleavage of the S-H bond ${ }^{17}$ and the formation of a Au-S bond, ${ }^{18}$ while AuNPs can be conveniently self-assembled on the thiol groups present in the network. MPTS sol-gel and AuNPs-based materials have been used for the immobilization of enzymes, such as dehydrogenase, ${ }^{17,19}$ bilirubin oxidase, ${ }^{20}$ lactate oxidase ${ }^{21}$ and glucose oxidase, ${ }^{22}$ for the development of biosensors. However, to the best of our present knowledge, a tyrosinasebased biosensor fabricated by AuNPs and sol-gel film has not been reported.

In the present study, the integration of AuNPs and MPTS derived three-dimensional network has been developed to immobilize tyrosinase for the detection of catechol. The MPTS sol-gel was modified on the electrode surface at first, and then AuNPs were conveniently self-assembled on the thiol groups present both inside and on the surface of the network..$^{23}$ Finally, the enzyme was immobilized on the surface of the modified electrode. Immobilizing the enzyme on the electrode surface is more conducive to the reaction of the enzyme and the substrate. It is expected that the AuNPs assembled inside the network might promote electron transfer between the electrode surface and the enzymatic reaction product, while the AuNPs on the 
surface of the network could increase the activity and immobilization amount of tyrosinase, and enhance the sensitivity of the biosensor. Desirable sensor performances, such as operational stability, reusability and sensitivity, could be expected.

\section{Experimental}

\section{Reagents and chemicals}

Chloroauric acid trihydrate $\left(\mathrm{HAuCl}_{4} \cdot 3 \mathrm{H}_{2} \mathrm{O}\right)$ and (3-mercaptopropyl)-trimethoxysilane were purchased from Sigma. Tyrosinase, obtained from Sigma, was used without further purification. Catechol, ascorbic acid (AA), acetaminophen (AP) and uric acid (UA) were purchased from Tianjin Kaitong Chemical Reagent Co., Ltd. (China). Potassium ferricyanide was the product of Shanghai Chemical Reagent Factory (Shanghai, China). Other chemicals were of analytical reagent grade, and used as received. All solutions were prepared with ultra-pure water.

\section{Apparatus}

Electrochemical measurements were carried out using a computer-controlled CHI 750C electrochemical work station (CH Instruments, Chenhua Co., Shanghai, China) at ambient temperature $\left(23 \pm 2{ }^{\circ} \mathrm{C}\right)$. A conventional three-electrode system was used. The working electrode was a tyr/AuNPs/MTPS/Au electrode, and the reference electrode was a $\mathrm{Ag} / \mathrm{AgCl}$ (3 M $\mathrm{KCl})$. The counter electrode was a platinum wire. The current-time data were recorded by applying a potential of $-0.18 \mathrm{~V}$ on a stirred cell. Electrochemical impedance spectroscopy (EIS) measurements were carried out in the presence of $5.0 \mathrm{mM} \mathrm{K}_{3}\left[\mathrm{Fe}(\mathrm{CN})_{6}\right] / \mathrm{K}_{4}\left[\mathrm{Fe}(\mathrm{CN})_{6}\right](1: 1)$ and $0.10 \mathrm{M}$ PBS (pH 7.0) by applying an $\mathrm{AC}$ voltage with $5 \mathrm{mV}$ amplitude in a frequency range from $5.0 \times 10^{-2}$ to $1.0 \times 10^{-5} \mathrm{~Hz}$.

Optical spectra of the prepared gold colloidal particles were obtained on an UV-vis spectrophotometer (Bejing Purking General Instrument Co., Ltd., China).

\section{Preparation of silica sol-gel}

Silica sol-gel was prepared ${ }^{24,25}$ by mixing MPTS, ethanol, and water (as $0.1 \mathrm{M} \mathrm{HCl}$ ) at a molar ratio of 1:3:3. The mixture was stirred vigorously for 30 min until a clear and homogeneous solution was obtained. The sol-gel obtained was stored at room temperature.

\section{Preparation of AuNPs}

All glass-wares used in the preparation of $\mathrm{Au}$ colloidal particles were cleaned in freshly prepared aqua regia $\left(\mathrm{HCl}: \mathrm{HNO}_{3}\right.$ $=3: 1(\mathrm{v} / \mathrm{v}))$, rinsed thoroughly with water, and oven-dried prior to use. Warning: aqua regia is a powerful oxidizing agent and it should be handled with extreme care. Au colloidal particles were prepared according to the literature. ${ }^{26} \mathrm{Au}$ colloidal particles of $6 \mathrm{~nm}$-diameter were prepared by adding $100 \mu \mathrm{L}$ of $1 \%$ sodium citrate solution and $45 \mu \mathrm{L}$ of $0.075 \%$ sodium borohydride to $25 \mathrm{ml}$ of a $0.01 \%$ (w/w) $\mathrm{HAuCl}_{4}$ solution, and stirring the solution at room temperature until a wine-red color was observed.

\section{Preparation of the tyr/AuNPs/MTPS/Au electrode}

A gold disk-electrode ( $2 \mathrm{~mm}$ in diameter) was polished with $1.0,0.3$ and $0.05 \mu \mathrm{m}$ alumina slurry, and then rinsed thoroughly with water in an ultrasonic bath between each polish step. Then the polished electrode was sonicated in water, absolute ethanol and water successively, and then the electrode was allowed to

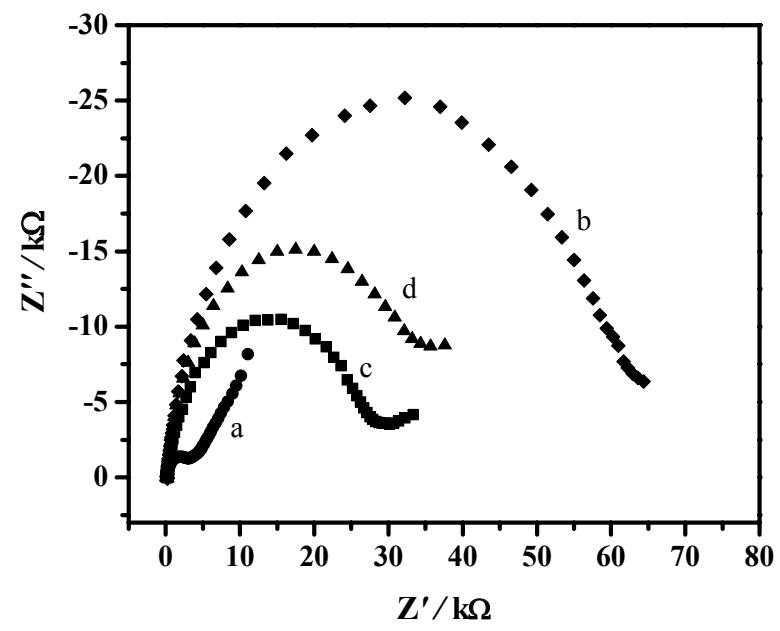

Fig. 1 Nyquist plot of various modified electrodes in the presence of $5.0 \mathrm{mM}\left[\mathrm{Fe}(\mathrm{CN})_{6}\right]^{3-} /\left[\mathrm{Fe}(\mathrm{CN})_{6}\right]^{4-}$ and $0.10 \mathrm{M} \mathrm{PBS}(\mathrm{pH} 7.0)$. The frequency range was $5.0 \times 10^{-2}$ to $1.0 \times 10^{-5} \mathrm{~Hz}$. (a) Bare Au electrode, (b) MPTS/Au electrode, (c) AuNPs/MPTS/Au electrode, (d) tyr/ AuNPs/MPTS/Au electrode.

dry at room temperature. The cleaned Au electrode was incubated in MPTS sol-gel at room temperature for $3 \mathrm{~h}$ for the spontaneous adsorption of the sol-gel network onto the electrode surface. Then, the electrode was rinsed in turn with ethanol and water. Subsequently, the electrode was exposured to a gold colloid solution for $5 \mathrm{~h}$ at $4{ }^{\circ} \mathrm{C}$ for the self-assembly of AuNPs on this three-dimensional silicate network. After that, a $5 \mu \mathrm{L}$ of $2.6 \mathrm{mg} \mathrm{mL}^{-1}$ tyrosinase solution was dropped onto the surface of the modified electrode, and kept in a refrigerator at $4{ }^{\circ} \mathrm{C}$ overnight. Finally, the enzyme electrode was immersed in $4 \mathrm{mM}$ PBS to wash out nonimmobilized enzyme, and then stored in PBS ( $\mathrm{pH} 7.0)$ at $4^{\circ} \mathrm{C}$ for future use. The resulting electrode is denoted as tyr/AuNPs/MTPS/Au in the text.

\section{Results and Discussion}

\section{EIS characterization of the modified electrodes}

EIS is an effective method for probing the interface properties of surface-modified electrodes. Figure 1 exhibits the Nyquist plots of the impedance spectroscopy of the bare and modified gold electrodes. The impedance spectrum includes a semicircle part, corresponding to the electron-transfer limited process, and a linear part, resulting from the diffusion-limited process. At a bare gold electrode, only a very small semicircle could be observed, showing a low transfer resistance (Fig. 1(a)). In the case of MPTS sol-gel film-modified electrode, the diameter of the semicircle increased greatly (Fig. 1(b)), implying that the layer of MPTS sol-gel film obstruct electron-transfer of the redox probe. The results indicate that the MPTS sol-gel film inhibited the penetration of the $\left[\mathrm{Fe}(\mathrm{CN})_{6}\right]^{3-} /\left[\mathrm{Fe}(\mathrm{CN})_{6}\right]^{4-}$ redox species toward the electrode. After self-assembling of AuNPs on a sol-gel network, the interfacial resistance decreased greatly (Fig. 1(c)), indicating that the AuNPs on sol-gel network promoted electron transfer between the electrode and the redox couple. After the assembling of tyrosinase on the surface of the electrode, the interfacial electron-transfer resistance increased (Fig. 1(d)). This result is ascribed to the non-conductive properties of tyrosinase, which hindered the access of the redox probe to the electrode, and obstructed electron-transfer. The 


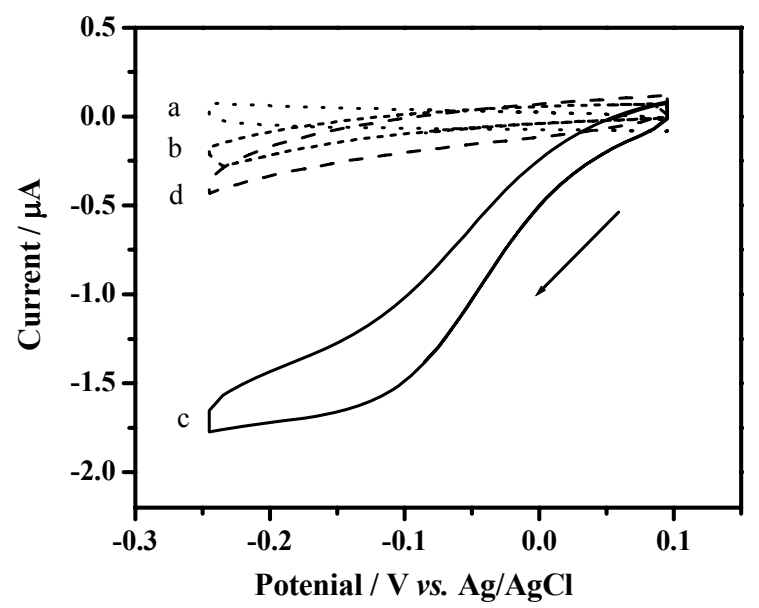

Fig. 2 Cyclic voltammograms at MPTS/Au electrode (a), tyr/MPTS/ Au electrode (b), tyr/AuNPs/MPTS/Au electrode (c), and AuNPs/ MPTS/Au electrode (d) in $10 \mathrm{mM}$ PBS (pH 7.0) containing $0.1 \mathrm{mM}$ catechol. Scan rate, $100 \mathrm{mV} \mathrm{s}^{-1}$.

results of EIS proved indirectly the assembly of the MPTS sol-gel film, AuNPs and tyrosinase on the surface of the electrode.

\section{Enhancement effect of AuNPs on electrocatalytic reaction of catechol}

In the present work, catechol was selected as the typical model of phenolic compounds to study the electrochemical characteristics of the resulting biosensor. Tyrosinase catalyzes the oxidation of catechols to o-quinones, which is electrochemically reduced back to catechol at the electrode. The cyclic voltammograms in the presence of catechol at differently modified electrodes are shown in Fig. 2. At a MPTS modified electrode (MPTS/Au, Fig. 2(a)), no peak current was found, which indicated that the catechol cannot be directly oxidated at the tyrosinase-free electrode in the potential from -0.25 to $+0.10 \mathrm{~V}$. At a tyrosinase-immobilized electrode (tyr/MPTS/Au, Fig. 2(b)), for which we believe tyrosinase was attached on MPTS by the van der Waals force or hydrogen bonding, no obvious reduction peak was observed, and only a small reduction current increase occurred, compared with the MPTS/Au electrode. This is attributed to a very weak reduction behavior of $o$-quinine liberated from the biocatalytic reaction, which indicated that the MPTS sol-gel film could not effectively form an electron-transfer channel, and obstructed the electron-transfer of the enzymatic reaction. However, in the case of a AuNPs-modified enzyme electrode (tyr/AuNPs/MPTS/ $\mathrm{Au}$ ), an obvious reduction peak was observed, starting around $-0.10 \mathrm{~V}$ (Fig. 2(c)), which is consistent with the phenol biosensor based on a photocurable membrane with tyrosinase. ${ }^{27}$ In order to investigate whether the reduction peak was from the immobilized AuNPs on the catalytic oxidation of catechol, AuNPs and MPTS modified electrode (tyrosinase-free) was prepared. It was found that no significant current response was observed at AuNPs/MPTS/Au (Fig. 2(d)), showing no obviously catalytic effect of the immobilized AuNPs in sol-gel matrix on the oxidation of catechol. It was obvious that the enhancement effect of AuNPs on the current response was also related to the enzymes. This result is in agreement with the case of the gold colloids and horseradish peroxidase-modified gold electrode. ${ }^{28}$

The AuNPs in three-dimensional network dramatically enhanced the electrochemical response of catechol at the tyr/

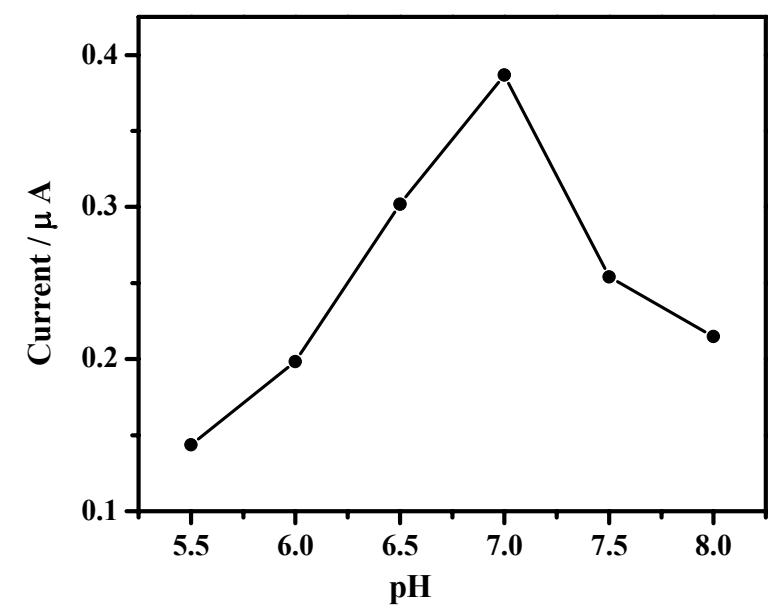

Fig. 3 Effect of the solution $\mathrm{pH}$ on the stable current response of the enzyme electrode in $0.10 \mathrm{M}$ PBS containing $0.1 \mathrm{mM}$ catechol. Applied potential, $-0.18 \mathrm{~V} v s$. $\mathrm{Ag} / \mathrm{AgCl}(3 \mathrm{M} \mathrm{KCl})$.

AuNPs/MPTS/Au electrode, resulting in an obviously increasing reduction current. This may be explained as follows. Gold particles of nanometer scale have some important size-dependent properties due to the quantum size effect. ${ }^{29}$ AuNPs show the electron injection effect and electron-withdraw effect ${ }^{30}$ and display unique electrical conductivity. In the resulting biosensor, AuNPs within the MPTS sol-gel three-dimensional network could effectively promote electron transfer between the surface of the electrode and the enzymatic reaction product. AuNPs assembled on the surface of the MPTS sol-gel film, as an electron acceptor, could adsorb electrons from the reactive center of enzymes, and accelerate them to return to the oxidized form, while AuNPs, as electron donors, could transfer electrons to oxygen, resulting in an obvious increase in the reactivity of tyrosinase. Therefore, AuNPs could mediate the electron transfer-reaction, and enhance the electrochemical reduction of the corresponding $o$-quinones.

On the other hand, the interaction between protein molecules and AuNPs is very strong due to the very high surface-tovolume ratio and high surface energy of AuNPs. ${ }^{31}$ Tyrosinase can be adsorbed onto the AuNPs, and retain their catalytic activity because of a microenvironment provided by AuNPs, similar to the native environment of redox proteins. ${ }^{32}$

\section{Effects of the applied potential and the solution $\mathrm{pH}$}

The effect of the applied potential on the biosensor response was investigated in the potential range from -0.3 to $0 \mathrm{~V}$ in a PBS containing $0.1 \mathrm{mM}$ catechol. The results showed that the response current gradually increased with a change of the applied potential from $0.0 \mathrm{~V}$ up to $-0.3 \mathrm{~V}$, and no maximum current response was obtained. However, the response currents of the electrode became obviously unstable for more negative potential values. Taking into account both the response sensitivity and the operational stability, a potential of $-0.18 \mathrm{~V}$ was selected for subsequent amperometric experiments.

The response behavior of the biosensor to $0.1 \mathrm{mM}$ catechol in PBS with different $\mathrm{pH}$ values, ranging from 5.5 to 8.0, was investigated at an applied potential of $-0.18 \mathrm{~V}$, and the results are shown in Fig. 3. It can be seen that the response current increased with the $\mathrm{pH}$ value ranging from 5.5 to 7.0 ; after reaching its maximum at $\mathrm{pH} 7.0$, it decreased as the $\mathrm{pH}$ increased further. The results show that the activity of the 


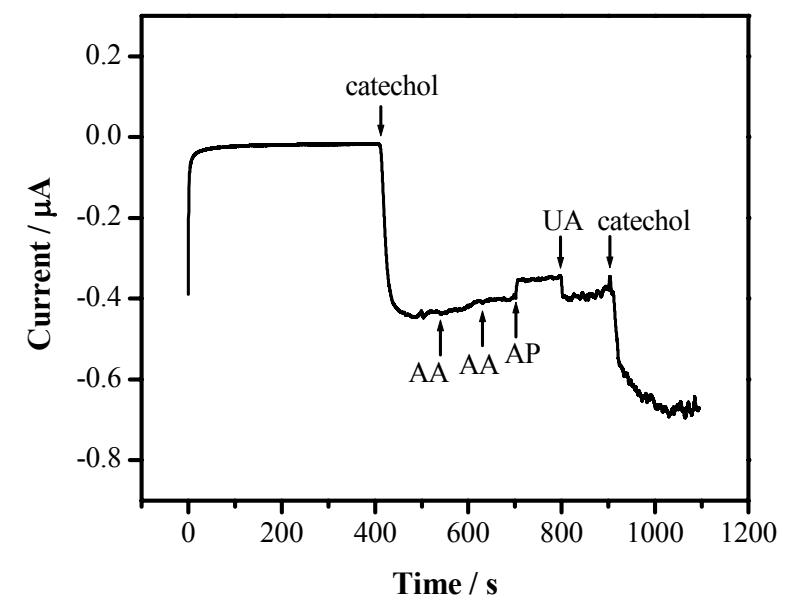

Fig. 4 Amperometric response obtained at the tyr/AuNPs/MTPS/Au electrode in 0.10 M PBS solution ( $\mathrm{pH} 7.0$ ) on successive injection of $0.05 \mathrm{mM}$ catechol, $0.10,0.20 \mathrm{mM}$ AA, $1 \mathrm{mM} \mathrm{AP}, 0.2 \mathrm{mM}$ UA, and then $0.05 \mathrm{mM}$ catechol. Applied potential, $-0.18 \mathrm{~V} v$ s. $\mathrm{Ag} / \mathrm{AgCl}(3 \mathrm{M}$ $\mathrm{KCl})$.

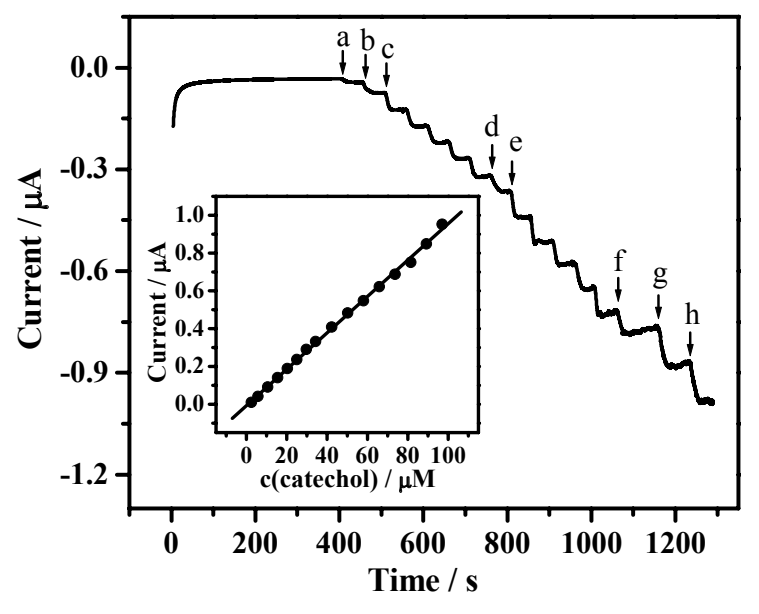

Fig. 5 Amperometric response of tyr/AuNPs/MTPS/Au electrode on the successive additions of catechol $(1.7 \mu \mathrm{M}$ (a), $3.3 \mu \mathrm{M}$ (b), $5.0 \mu \mathrm{M}(\mathrm{c}-\mathrm{d}), 8.3 \mu \mathrm{M}(\mathrm{e}-\mathrm{f})$ and $15.9 \mu \mathrm{M}(\mathrm{g}, \mathrm{h}))$ to $10 \mathrm{mM}$ PBS (pH 7.0). Inset, dynamic response curve for catechol. Applied potential, $-0.18 \mathrm{~V} v s . \mathrm{Ag} / \mathrm{AgCl}(3 \mathrm{M} \mathrm{KCl})$.

Table 1 Analytical characteristics of tyrosinase-based biosensors for catechol

\begin{tabular}{lccccccc}
\hline \multicolumn{1}{c}{ Electrode } & Linear range/ $\mu \mathrm{M}$ & $r$ & Applied potential $/ \mathrm{V}$ & Response time/s & LOD/ $\mu \mathrm{M}$ & RSD, $\%$ & Ref. \\
\hline AuNPs/graphite-Teflon & $0.010-8.0$ & 0.997 & -0.1 & - & 0.003 & $6.8, n=5$ \\
AuNPs/GCE & $0.5-50$ & 0.9998 & -0.1 & - & 0.15 & $3.6, n=6$ & 33 \\
Graphite-Teflon & $0.15-15$ & 0.997 & -0.15 & - & 0.1 & $4.0, n=10$ & 35 \\
MWCNT/Nafion/GCE & $1-23$ & 0.997 & -0.1 & 8 & 0.22 & - & 36 \\
PTS-PPY/GCP & $5.6-74.3$ & 0.999 & 0.05 & 40 & 1.5 & - & 37 \\
AuNPs/MPTS/Au & $1.7-96$ & 0.9992 & -0.18 & 8 & 0.56 & $3.2, n=10 \quad$ This work \\
\hline
\end{tabular}

GCE, glassy carbon electrode; MWCNT, multiwalled carbon nanotube; PTS, para-toluene sulfonate; PPY, polypyrrole; GCP, Glassy carbon plate.

a. Measured vs. $\mathrm{Ag} / \mathrm{AgCl}$ reference electrode.

enzyme is greatly influenced by the solution $\mathrm{pH}$ value. This can be explained as follows. Enzymes, like all proteins, are folded into three-dimensional shapes (tertiary structure). The charge of its component amino acids changes with the change in the $\mathrm{pH}$ value. When the $\mathrm{pH}$ value becomes very high or too low, then the basic structure of the enzyme undergoes changes. As a result, the active site of the enzyme is changed, and then fails to bind well with the substrate. In order to achieve the maximum sensitivity, a PBS of pH 7.0 was selected as the optimal supporting electrolyte in subsequent experiments.

\section{Effect of electroactive interference}

The current responses to the biosensor upon the injection of catechol and various electroactive interferences at the applied potential of $-0.18 \mathrm{~V}$ are shown in Fig. 4. It could be found that upon the addition of $0.20 \mathrm{mM} \mathrm{AA}, 1 \mathrm{mM}$ AP and $0.2 \mathrm{mM} \mathrm{UA}$, the observed current responses were very small, in contrast to the large response to the addition of $0.05 \mathrm{mM}$ catechol. This demonstrates that the tyr/AuNPs/MTPS/Au electrode has good anti-interferent ability.

\section{Amperometric response of the biosensor toward catechol}

The amperometric response of the tyr/AuNPs/MTPS/Au electrode with successive additions of catechol to $10 \mathrm{mM}$ PBS (pH 7.0) at an applied potential of $-0.18 \mathrm{~V}$ is shown in Fig. 5. As can be seen, the tyr/AuNPs/MTPS/Au electrode exhibited a rapid and sensitive response to changes in the concentration of catechol, reaching $95 \%$ of the steady-state current within $8 \mathrm{~s}$. Under the optimum conditions, the response current is linear to the catechol in the concentration range from 1.7 to $96 \mu \mathrm{M}$ with a correlation coefficient of 0.9992 (Fig. 5, inset). From the

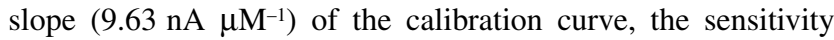
was calculated to be $306.7 \mathrm{~mA} \mathrm{M}^{-1} \mathrm{~cm}^{-2}$, with an amperometric detection limit of $0.56 \mu \mathrm{M}$. A relative standard deviation (RSD) of $3.5 \%$ has been observed for ten successive determination of $0.1 \mathrm{mM}$ catechol, indicating the reliable reproducibility of the sensor. The analytical performance of the tyrosinase-based biosensor was compared with those reported for other recent tyrosinase-based biosensor, ${ }^{33-37}$ as can been seen in Table 1. It can be deduced that the developed tyr/AuNPs/MTPS/Au biosensor allows a wide range of linearity, and provides a fast current response.

\section{Conclusions}

An electrochemical biosensor for the detection of phenolic compounds was developed by integrating the tyrosinase and AuNPs with the three-dimensional networks of MPTS. The tyrosinase and AuNPs was efficiently immobilized on a three-dimensional networks-modified gold electrode. The presence of AuNPs in the MPTS sol-gel film promoted electron transfer between the electrode surface and the enzymatic reaction product. In addition, AuNPs on the surface of the 
MPTS sol-gel film significantly affected the electron-transfer kinetics of the enzyme reaction, and enhanced the electrochemical reduction of the corresponding $o$-quinones. The resulting biosensor provided a suitable microenvironment for retaining the bioactivity of tyrosinase and exhibited a good analytical performance for the amperometric detection of catechol.

\section{Acknowledgements}

This work was financially supported by the Natural Science Foundation of Hebei Province of China (No. B2010000844) and Research Foundation of Education Department of Hebei Province of China (No. ZH2012078).

\section{References}

1. M. Sánchez-Paniagua López, F. Tamimi, E. LópezCabarcos, and B. López-Ruiz, Biosens. Bioelectron., 2009, 24, 2574.

2. S. Wang, Y. Tan, D. Zhao, and G. Liu, Biosens. Bioelectron., 2008, 23, 1781.

3. J. Zhang, J. Lei, Y. Liu, J. Zhao, and H. Ju, Biosens. Bioelectron., 2009, 24, 1858.

4. V. Carralero, M. L. Mena, A. Gonzalez-Cortés, P. YáñezSedeño, and J. M. Pingarrón, Biosens. Bioelectron., 2006, 22, 730 .

5. C. Apetrei, M. L. Rodríguez-Méndez, and J. A. D. Saja, Electrochim. Acta, 2011, 56, 8919.

6. Q. Ameer and S. B. Adeloju, Sens. Actuators, B, 2009, 140, 5.

7. Q. Zhang, Y. Qu, X. Zhang, J. Zhou, and H. Wang, Biosens. Bioelectron., 2003, 19, 509.

8. H. Zejli, J. L. Hidalgo-Hidalgo de Cisneros, I. NaranjoRodriguez, B. Liu, K. R. Temsamani, and J. L. Marty, Anal. Chim. Acta, 2008, 612, 198.

9. X. Ji, X. Li, N. Wang, R. Ni, X. Liu, and H. Xiong, Chin. Chem. Lett., 2010, 21, 1239.

10. Y. Wei, H. Dong, J. Xu, and Q. Feng, ChemPhysChem, 2002, 3, 802 .

11. C. Shen and N. M. Kostic, J. Am. Chem. Soc., 1997, 119, 1304.

12. S. A. Ansari and Q. Husain, Biotechnol. Adv., 2012, 30, 512.

13. S. Tokonami, Y. Yamamoto, H. Shiigi, and T. Nagaoka, Anal. Chim. Acta, 2012, 716, 76.

14. A. K. Yagati, T. Lee, J. Min, and J. Choi, Colloids Surf., B,
2012, 92, 161.

15. Y. Fang, S. Guo, C. Zhu, Y. Zhai, and E. Wang, Langmuir, 2010, 26, 11277

16. C. Shan, H. Yang, D. Han, Q. Zhang, A. Ivaska, and L. Niu, Biosens. Bioelectron., 2010, 25, 1070.

17. B. K. Jena and C. R. Raj, Anal. Chem., 2006, 78, 6332.

18. J. B. Schlenoff, M. Li, and H. Ly, J. Am. Chem. Soc., 1995, 117, 12528.

19. L. Deng, L. Zhang, L. Shang, S. Guo, D. Wen, F. Wang, and S. Dong, Biosens. Bioelectron., 2009, 24, 2273.

20. P. Kannan, H. Chen, V. T. Lee, and D. Kim, Talanta, 2011, 86,400

21. A. M. Parra-Alfambra, E. Casero, M. D. Petit-Domínguez, M. Barbadillo, F. Pariente, L. Vázquez, and E. Lorenzo, Analyst, 2011, 136, 340.

22. S. Tuncagil, C. Ozdemir, D. O. Demirkol, S. Timur, and L. Toppare, Food Chem., 2011, 127, 1317.

23. S. Bharathi, M. Nogami, and S. Ikeda, Langmuir, 2001, 17, 1.

24. G. Maduraiveeran and R. Ramaraj, J. Electroanal. Chem., 2007, 608, 52.

25. Y. Yang, T. Tseng, J. Yeh, C. Chen, and S. Lou, Sens. Actuators, B, 2008, 131, 533

26. K. R. Brown, A. P. Fox, and M. J. Natan, J. Am. Chem. Soc., 1996, 118, 1154

27. S. A. Hanifah, L. Y. Heng, and M. Ahmad, Sensors, 2008, 8, 6407.

28. D. Lan, B. Li, and Z. Zhang, Biosens. Bioelectron., 2008 , 24, 934.

29. X. Y. Chen, J. R. Li, X. C. Li, and L. Jiang, Biochem. Biophys. Res. Commun., 1998, 245, 352.

30. L. Jiang, Chin. J. Inorg. Chem., 2000, 16, 185.

31. M. A. Zaitoun, Spectroscopy, 2005, 19, 119.

32. A. L. Crumbliss, S. C. Perine, J. Stonehuerner, K. R. Tubergen, J. G. Zhao, R. W. Henkens, and J. P. O’Daly, Biotechnol. Bioeng., 1992, 40, 483.

33. V. Carralero, M. L. Mena, A. Gonzalez-Cortés, P. YáñezSedeño, and J. M. Pingarrón, Biosens. Bioelectron., 2006 , 22,730

34. V. C. Sanz, M. L. Mena, A. Gonzalez-Cortés, P. YáñezSedeño, and J. M. Pingarrón, Anal. Chim. Acta, 2005, 528 , 1 .

35. B. Serra, S. Jiménez, M. L. Mena, A. J. Reviejo, and J. M. Pingarrón, Biosens. Bioelectron., 2002, 17, 217.

36. Y. Tsai and C. Chiu, Sens. Actuators, B, 2007, 125, 10.

37. Rajesh, W. Takashima, and K. Kaneto, React. Funct. Polym., 2004, 59, 163. 